\title{
Efficacy and safety of a switch to latanoprost $0.005 \%$ + timolol maleate $0.5 \%$ fixed combination eyedrops from latanoprost $0.005 \%$ monotherapy
}

This article was published in the following Dove Press journal:

Clinical Ophthalmology

19 May 2012

Number of times this article has been viewed

\section{Kenji Inoue' \\ Takayuki Fujimoto' \\ Risako Higa' \\ Ryo Moriyama' \\ Hiromi Kohmoto' \\ Haruka Nagumo' \\ Masato Wakakura' \\ Goji Tomita²}

'Inouye Eye Hospital, Chiyoda-ku, Tokyo, ${ }^{2}$ Second Department of Ophthalmology, Toho University School of Medicine, Meguro-ku, Tokyo, Japan
Correspondence: Kenji Inoue Inouye Eye Hospital, 4-3 KandaSurugadai, Chiyoda-ku,

Tokyo 101-0062, Japan

Tel +8I3 329509 I I

Fax +8I 332950917

Email inoue-k@inouye-eye.or.jp
Background: The purpose of this prospective study was to investigate the intraocular pressure (IOP)-lowering effect and safety of latanoprost $0.005 \%$ + timolol maleate $0.5 \%$ fixed combination eyedrops, now available in Japan.

Materials and methods: Thirty-one patients diagnosed with primary open-angle glaucoma who had an insufficient intraocular pressure (IOP) decrease with latanoprost $0.005 \%$ eyedrop monotherapy were enrolled. The latanoprost $0.005 \%$ eyedrops were discontinued, and administration of latanoprost $0.005 \%$ /timolol maleate $0.5 \%$ fixed combination eyedrops was initiated without any washout period. IOP was compared before and at months 1, 3, and 6 after the switch. The incidence of adverse reactions was investigated at every follow-up visit.

Results: Mean IOP was $17.3 \pm 2.7 \mathrm{mmHg}$ before the switch, $15.5 \pm 2.6 \mathrm{mmHg}$ one month after the switch, $14.9 \pm 2.4 \mathrm{mmHg} 3$ months after the switch, and $15.1 \pm 2.2 \mathrm{mmHg} 6$ months after the switch, indicating that IOP decreased significantly after the change. The IOP reduction rate was $9.9 \% \pm 11.5 \%$ after one month, $13.1 \% \pm 10.9 \%$ after 3 months, and $11.2 \% \pm 11.8 \%$ after 6 months. Two patients $(6.5 \%)$ discontinued therapy due to adverse reactions (one case each of itchiness and bradycardia).

Conclusion: When latanoprost $0.005 \%$ eyedrop monotherapy was replaced by latanoprost $0.005 \%$ + timolol maleate $0.5 \%$ fixed combination eyedrops, IOP decreased significantly without increasing the frequency of administration, and safety was satisfactory.

Keywords: latanoprost $0.005 \%$, timolol maleate $0.5 \%$, fixed combination, eyedrops, intraocular pressure, switch

\section{Introduction}

The goal of treatment of patients diagnosed with glaucoma is preservation of visual function. Intraocular pressure (IOP)-lowering therapy is the only alternative that shows evidence of adequate preservation of the visual field. ${ }^{1,2}$ Generally, medical treatment is the first approach to decreasing IOP. Prostaglandin-related eyedrops are the primary therapy due to their powerful IOP-decreasing effects, their low incidence of systemic side effects, and their convenient once-daily administration. However, in cases where the IOP decrease is insufficient with monotherapy, a switch to another medication or initiation of adjunctive therapy is necessary. In multidrug cases where the frequency of administration increases, decreased adherence is a problem, ${ }^{3}$ so the most suitable option is once-daily administration of fixed combination eyedrops. In Japan, the latanoprost $0.005 \%$ + timolol maleate $0.5 \%$ fixed combination eyedrop (Xalacom ${ }^{\circledR}$; Pfizer Japan Inc, Japan, Tokyo) formulation was released in April 2010. 
The present study involved patients with primary open-angle glaucoma who were initially treated with latanoprost $0.005 \%$ eyedrop monotherapy. We investigated the IOPdecreasing effect and safety of a switch to latanoprost $0.005 \%$ + timolol maleate $0.5 \%$ fixed combination eyedrops.

\section{Materials and methods}

This study included 31 eyes from 31 patients (12 men, 19 women) diagnosed with primary open-angle glaucoma (including normal tension glaucoma) and treated at Inouye Eye Hospital between May 2010 and June 2011. The patients were using latanoprost monotherapy and had inadequate IOP control. Their mean age was $63.0 \pm 12.4$ (range 31-79) years. Twenty-one patients had primary open-angle glaucoma and 10 had normal tension glaucoma. The mean deviation in Humphrey visual field was $-7.2 \pm 5.9$ (range $-27.46-1.45$ ) dB. The mean IOP before switching to latanoprost $0.005 \%+$ timolol maleate $0.5 \%$ fixed combination eyedrops was $17.3 \pm 2.7$ (range 12-23) $\mathrm{mmHg}$. If both eyes met the eligibility criteria, the eye with the higher IOP was selected; if the IOP was equivalent in both eyes, the right eye was selected for treatment. In a monocular case, the affected eye was used for analysis.

The patients discontinued latanoprost $0.005 \%$ eyedrops and switched to latanoprost $0.005 \%+$ timolol maleate $0.5 \%$ fixed combination eyedrops (once daily at night) without any washout period. Eyedrops were administered at the same time of the day, and IOP was measured before and at 1, 3, and 6 months after the switch by the same examiner using a Goldmann applanation tonometer at the same time of day. IOP before and at 1, 3, and 6 months after the switch was compared by analysis of variance and Bonferroni/Dunnett's tests. IOP reduction at 1, 3 and 6 months after the switch was calculated and compared. After the switch, the incidence of adverse reactions was investigated at every follow-up visit. The statistical significance level in all cases was $P<0.05$. The study was approved by the ethics committee of Inouye Eye Hospital, and conducted according to the Declaration of Helsinki after explaining the purpose and details of the study to the patients and obtaining informed consent in writing. Institutional review board approval was sought before any procedures were performed.

\section{Results}

The mean IOP was $15.5 \pm 2.6 \mathrm{mmHg}$ one month after the switch, $14.9 \pm 2.4 \mathrm{mmHg} 3$ months after the switch, and $15.1 \pm 2.2 \mathrm{mmHg} 6$ months after the switch. There was a significant decrease in IOP compared with the mean baseline value of $17.3 \pm 2.7 \mathrm{mmHg}$ before the switch $(P<0.0001$, Figure 1). At 6 months after the switch, IOP had decreased by more than $2 \mathrm{mmHg}$ in 17 cases $(60.7 \%)$ and was within $1 \mathrm{mmHg}$ in 11 cases (39.3\%, Figure 2). Mean IOP reduction was $1.8 \pm 2.2 \mathrm{mmHg}$ one month after the switch, $2.4 \pm 2.2 \mathrm{mmHg} 3$ months after the switch, and $2.1 \pm 2.3 \mathrm{mmHg} 6$ months after the switch, which was not significantly different $(P=0.6408)$. The mean IOP reduction rate was $9.9 \% \pm 11.5 \%$ one month after the switch, $13.1 \% \pm 10.9 \% 3$ months after the switch, and $11.2 \% \pm 11.8 \% 6$ months after the switch, which was not significantly different $(P=0.5682)$.

Adverse reactions appeared in two cases (6.5\%), including one case each of itching and bradycardia.

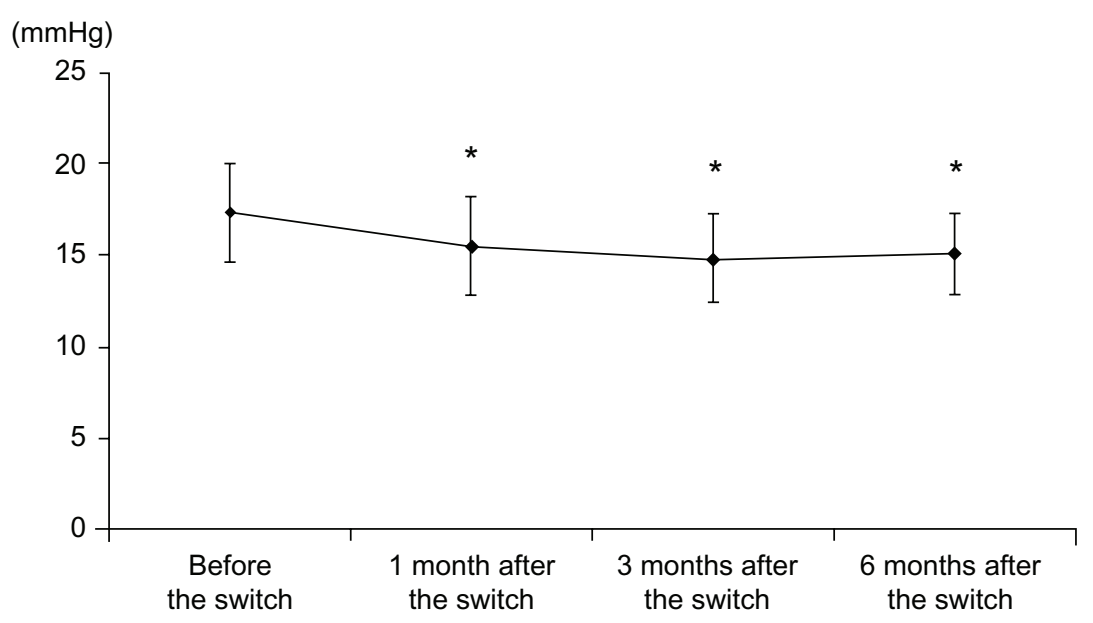

Figure I Intraocular pressure before and after the switch to the fixed combination of latanoprost and timolol maleate. Notes: Analysis of variance, Bonferroni/Dunnett's test, $* P<0.0001$. 


\section{6 months after the switch}

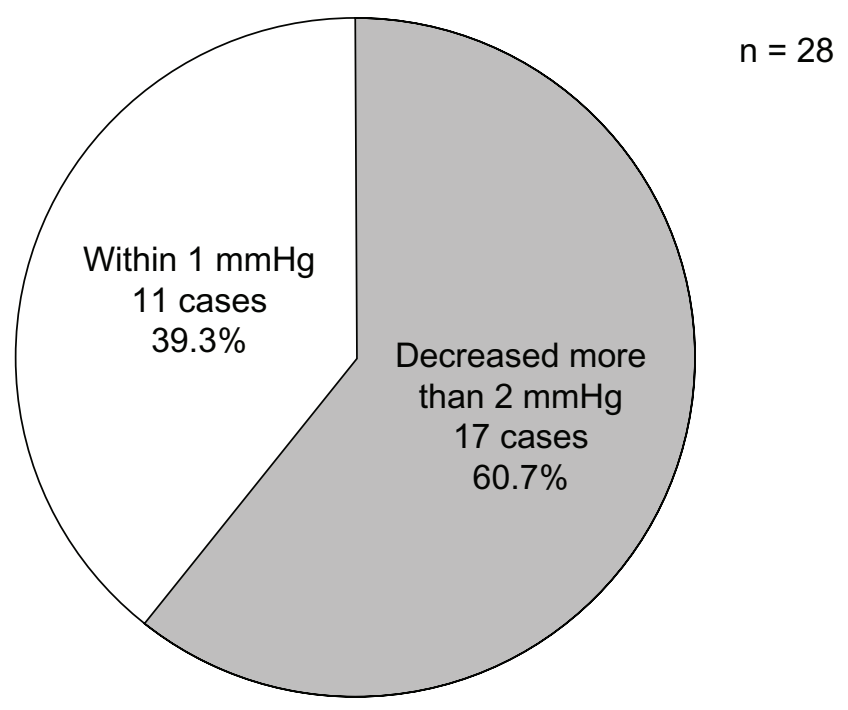

Figure 2 Reductions in intraocular pressure 6 months after the switch to the fixed combination of latanoprost and timolol maleate.

After discontinuation of the latanoprost $0.005 \%+$ timolol maleate $0.5 \%$ combination, the symptoms immediately disappeared. One patient discontinued as a result of stopping coming to the hospital after 5 months.

\section{Discussion}

If the decrease in IOP is insufficient with latanoprost $0.005 \%$ eyedrop monotherapy, $\beta$-blocker or carbonic anhydrase inhibitor eyedrops can be added to the regimen, or medication can be switched to fixed combination eyedrops. When fixed combination eyedrops are used instead of combined therapy, the administration frequency decreases, adherence improves, and IOP may decrease. The administration frequency for latanoprost $0.005 \%$ + timolol maleate $0.5 \%$ fixed combination eyedrops is once daily, but the original administration frequency for timolol maleate $0.5 \%$ eyedrops is twice daily, so there is a concern that there could be a reduction in efficacy. Thus, it is of interest whether the IOP decrease associated with latanoprost $0.005 \%$ + timolol maleate $0.5 \%$ fixed combination eyedrop monotherapy is equal to that associated with concomitant use of latanoprost $0.005 \%$ and timolol maleate $0.5 \%$ eyedrops. We have previously reported on hypotensive efficacy in 162 patients diagnosed with primary open-angle glaucoma or ocular hypertension who were switched from concomitant use of latanoprost $0.005 \%$ eyedrops and timolol maleate $0.5 \%$ eyedrops to latanoprost $0.005 \%$ + timolol maleate $0.5 \%$ fixed combination eyedrops. ${ }^{4}$ The mean IOP was $15.2 \pm 3.3 \mathrm{mmHg}$ before the switch and $15.1 \pm 3.2 \mathrm{mmHg} 3$ months after the switch, indicating that there was no significant difference in efficacy. Diestelhorst et al randomly assigned patients to latanoprost $0.005 \%+$ timolol maleate $0.5 \%$ fixed combination eyedrops ( 255 cases) or use of latanoprost $0.005 \%$ eyedrops and timolol maleate $0.5 \%$ eyedrops concomitantly (247 cases) in a double-blind study among patients diagnosed with primary open-angle glaucoma, ocular hypertension, pigmentary glaucoma, or exfoliation glaucoma. ${ }^{5}$ After 12 weeks of administration, IOP reduction associated with the latanoprost $0.005 \%+$ timolol maleate $0.5 \%$ fixed combination eyedrop monotherapy group was $8.7 \pm 0.2 \mathrm{mmHg}$, and that of the concomitant latanoprost $0.005 \%$ eyedrops and timolol maleate $0.5 \%$ eyedrops group was $9.0 \pm 0.2 \mathrm{mmHg}$, indicating that there was no significant difference between the groups. However, adverse reactions appeared significantly less often among patients in the latanoprost $0.005 \%$ + timolol maleate $0.5 \%$ fixed combination eyedrop monotherapy group $(P<0.05)$. Findings from a switching study ${ }^{4}$ and a double-masked study ${ }^{5}$ have indicated that the IOP decrease appears to be identical for patients who use latanoprost $0.005 \%$ and timolol maleate $0.5 \%$ eyedrops concomitantly and those who use latanoprost $0.005 \%$ + timolol maleate $0.5 \%$ fixed combination eyedrop monotherapy.

There have been many reports of hypotensive effects following a switch from latanoprost $0.005 \%$ eyedrop monotherapy to latanoprost $0.005 \%$ + timolol maleate $0.5 \%$ fixed combination eyedrops. ${ }^{6-9}$ Kitazawa et al administered latanoprost $0.005 \%$ eyedrops for 4 weeks to 289 patients with primary open-angle glaucoma and ocular hypertension. ${ }^{6}$ Afterwards, they divided the patients into latanoprost $0.005 \%$ + timolol maleate $0.5 \%$ fixed combination eyedrops and latanoprost $0.005 \%$ monotherapy groups, and continued treatment for a further 8 weeks. In the latanoprost $0.005 \%+$ timolol maleate $0.5 \%$ fixed combination eyedrops group, mean IOP was $19.62 \pm 2.60 \mathrm{mmHg}$ before the switch, but was decreased to $17.03 \pm 2.67 \mathrm{mmHg} 8$ weeks after the switch. Mean IOP reduction was $2.59 \pm 2.40 \mathrm{mmHg}$ and the IOP reduction rate was $12.7 \% \pm 12.1 \%$. Dunker et al evaluated IOP among 51 patients with either exfoliation glaucoma, ocular hypertension, or pigmentary glaucoma for 6 months after an identical switch. ${ }^{7}$ There was a significant decrease in mean IOP 6 months after the switch $(17.7 \pm 3.6 \mathrm{mmHg})$ compared with beforehand $(20.7 \pm 3.6 \mathrm{mmHg})$. Mean IOP reduction was $2.9 \pm 3.6 \mathrm{mmHg}$. Hamacher et al evaluated the IOP of 69 patients with exfoliation glaucoma, ocular hypertension, or pigmentary glaucoma for more than 2 months after the same switch. ${ }^{8}$ The IOP after the switch $(17.8 \pm 2.9 \mathrm{mmHg})$ was significantly decreased compared 
with that beforehand $(20.8 \pm 3.4 \mathrm{mmHg})$. Mean IOP reduction was $3.1 \pm 3.6 \mathrm{mmHg}$. Polo et al evaluated IOP among 33 primary open-angle glaucoma or exfoliative glaucoma patients for 3 months after an identical switch. ${ }^{9}$ Mean IOP one month after the switch $(14.90 \pm 1.86 \mathrm{mmHg})$ and 3 months after the switch $(15.66 \pm 1.91 \mathrm{mmHg})$ was significantly decreased compared with beforehand $(20.38 \pm 5.33 \mathrm{mmHg})$. Mean IOP reduction value and IOP reduction rate were $5.48 \mathrm{mmHg}$ and $23.5 \%$, respectively, one month after the switch, and $4.71 \mathrm{mmHg}$ and $19.5 \%$, respectively, 3 months after the switch. The mean IOP reduction and IOP reduction rate was $1.8-2.3 \mathrm{mmHg}$ and $10.3 \%-12.6 \%$, respectively, in the present study, which is a little lower than the values reported previously. ${ }^{6-9}$ This is probably because the mean IOP before the switch was a little lower in the present study $(17.3 \pm 2.7 \mathrm{mmHg})$ compared with previous reports (19.6-20.8 mmHg). ${ }^{6-9}$

Hypotensive effects have previously been reported when a $\beta$-blocker is added to latanoprost therapy. ${ }^{10,11}$ Miura et al administered timolol maleate $0.5 \%$ eyedrops as adjunctive therapy for 12 weeks to 15 primary openangle glaucoma or ocular hypotension patients already receiving latanoprost $0.005 \%$ eyedrop monotherapy. ${ }^{10}$ IOP 12 weeks after initiation of the adjunctive therapy was significantly decreased $(15.8 \pm 3.2 \mathrm{mmHg})$ compared with baseline $(18.5 \pm 3.7 \mathrm{mmHg})$, and mean IOP reduction was $2.7 \pm 2.3 \mathrm{mmHg}$. Liu et al administered $0.5 \%$ timolol gel-forming solution as an adjunctive therapy for 8 weeks to 26 patients who were diagnosed with either glaucoma or ocular hypertension. ${ }^{11}$ IOP after 8 weeks of adjunctive therapy $(15.4 \pm 2.3 \mathrm{mmHg})$ was also significantly decreased compared with baseline $(23.0 \pm 1.8 \mathrm{mmHg})$. Mean IOP reduction was $6.2 \pm 2.8 \mathrm{mmHg}$, and the mean IOP reduction rate was $27.0 \%$. When comparing the present study with previous studies ${ }^{10,11}$ of adjunctive therapy using timolol maleate $0.5 \%$ eyedrops, mean IOP reduction was $1.8-2.3 \mathrm{mmHg}$ in the present study and so a little lower than the $1.9-6.2 \mathrm{mmHg}$ observed in the previous studies. ${ }^{10,11}$ IOP before the switch in the present study $(17.3 \pm 2.7 \mathrm{mmHg})$ was a little lower than IOP before initiation of adjunctive therapy reported previously $(17.3-23.0 \mathrm{mmHg}){ }^{10,11}$ It is thought that IOP reduction is almost identical when latanoprost $0.005 \%$ eyedrops are switched to either latanoprost $0.005 \%+$ timolol maleate $0.5 \%$ fixed combination eyedrops or concomitant use of latanoprost $0.005 \%$ eyedrops and timolol maleate $0.5 \%$ eyedrops.

Adverse reactions associated with latanoprost $0.005 \%+$ timolol maleate $0.5 \%$ fixed combination eyedrops include hyperemia, stimulation, foreign body sensation, conjunctivitis, and keratitis. ${ }^{4-10}$ No serious adverse reactions have been reported previously, ${ }^{4-10}$ and none were observed in the present study. The latanoprost $0.005 \%+$ timolol maleate $0.5 \%$ fixed combination eyedrops contains a $\beta$-blocker, but blood pressure and pulse rate were not evaluated in the present study. One case of unexpected bradycardia was identified because the patient's blood pressure and pulse were regularly checked. Systemic adverse reactions, such as those affecting the cardiovascular and respiratory systems, can be induced by $\beta$-blockers, so careful patient monitoring is required.

The weaknesses of the present study are that the number of cases was small $(\mathrm{n}=31)$, it was not blinded, and it did not include a control group. Moreover, when switching eyedrops, there is the possibility that adherence improves, resulting in a IOP decrease. Because there were only 31 subjects, the spread of IOP and standard deviation became large. In conclusion, when latanoprost $0.005 \%$ eyedrop monotherapy was switched to latanoprost $0.005 \%+$ timolol maleate $0.5 \%$ fixed combination eyedrops in Japanese patients with primary open-angle glaucoma, mean IOP decreased significantly without an increase in frequency of administration, and safety was satisfactory. Nevertheless, when $\beta$-blockers are added to a therapeutic regimen, careful attention must be paid to the incidence of systemic adverse reactions, in particular those affecting the cardiovascular or respiratory systems.

\section{Disclosure}

The authors report no conflicts of interest in this work.

\section{References}

1. Collaborative Normal-Tension Glaucoma Study Group. The effectiveness of intraocular pressure reduction in the treatment of normal-tension glaucoma. Am J Ophthalmol. 1998;126:498-505.

2. The AGIS Investigators. The Advanced Glaucoma Intervention Study (AGIS): 7. The relationship between control of intraocular pressure and visual field deterioration. Am J Ophthalmol. 2000;130:429-440.

3. Djafari F, Lesk MR, Harasymowycz PJ, et al. Determinants of adherence to glaucoma medical therapy in a long-term patient population. J Glaucoma. 2009;18:238-242.

4. Inoue K, Okayama R, Higa R, et al. Ocular hypotensive effects and safety over three months of switching from an unfixed combination to latanoprost $0.005 \%$ /timolol maleate $0.5 \%$ fixed combination. $J$ Ocul Pharmacol Ther. 2011;27:581-587.

5. Diestelhorst M, Larsson LI; European-Canadian Latanoprost Fixed Combination Study Group. A 12-week, randomized, double-masked, multicenter study of the fixed combination of latanoprost and timolol in the evening versus the individual components. Ophthalmology. 2006;113:70-76.

6. Kitazawa Y; KP2035 Study Group. Phase III double-blind study of latanoprost/timolol combination (KP2035) in patients with primary open-angle glaucoma or ocular hypertension. Rinsho Ganka. 2009;63: 807-815. Japanese. 
7. Dunker S, Schmucker A, Maier H, et al. Tolerability, quality of life, and persistency of use in patients with glaucoma who are switched to the fixed combination of latanoprost and timolol. Adv Ther. 2007;24:376-386.

8. Hamacher T, Schinzel M, Schölzel-Klatt A, et al. Short term efficacy and safety in glaucoma patients changed to the latanoprost $0.005 \% /$ timolol maleate $0.5 \%$ fixed combination from monotherapies and adjunctive therapies. Br J Ophthalmol. 2004;88:1295-1298.

9. Polo V, Larrosa JM, Ferreras A, et al. Effect on diurnal intraocular pressure of the fixed combination of latanoprost $0.005 \%$ and timolol $0.5 \%$ administered in the evening in glaucoma. Ann Ophthalmol. 2008;40: $157-162$.
10. Miura $\mathrm{K}$, Ito $\mathrm{K}$, Okawa $\mathrm{C}$, et al. Comparison of ocular hypotensive effect and safety of brinzolamide and timolol added to latanoprost. J Glaucoma. 2008;17:233-237.

11. Liu JHK, Medeiros FA, Slight JR, et al. Comparison diurnal and nocturnal effects of brizolamide and timolol on intraocular pressure in patients receiving latanoprost monotherapy. Ophthalmology. 2009;116:449-454.
Clinical Ophthalmology

\section{Publish your work in this journal}

Clinical Ophthalmology is an international, peer-reviewed journal covering all subspecialties within ophthalmology. Key topics include: Optometry; Visual science; Pharmacology and drug therapy in eye diseases; Basic Sciences; Primary and Secondary eye care; Patient Safety and Quality of Care Improvements. This journal is indexed on

Submit your manuscript here: http://www.dovepress.com/clinical-ophthalmology-journal

\section{Dovepress}

PubMed Central and CAS, and is the official journal of The Society of Clinical Ophthalmology (SCO). The manuscript management system is completely online and includes a very quick and fair peer-review system, which is all easy to use. Visit http://www.dovepress.com/ testimonials.php to read real quotes from published authors. 